\title{
Biotechnology and Bioengineering Tools in COVID-19 Diagnostics - CRISPR and Beyond
}

\author{
Rwik Sen* \\ Department of Biomedical Engineering, Active Motif, USA.
}

*Corresponding author: Rwik Sen, Department of Biomedical Engineering, Active Motif, USA.
Received Date: December 11, 2020

Published Date: December 22, 2020

\section{Mini Review}

Advances in bioengineering and biotechnology are an urgent need of the hour because of the ongoing pandemic of COVID-19. Coronavirus disease 2019 or COVID-19 is caused by SARS-CoV-2 which is a strain of a coronavirus called SARS-CoV or Severe Acute Respiratory Syndrome Corona Virus. Globally, COVID-19 has claimed lives $1,575,810$ and infected $69,239,671$ people. In this direction, biotechnological and bioengineering endeavors have shown several promising results to control the pandemic, which are discussed in this mini review.

Worldwide governments are aiming for COVID-19 testing to available on a large-scale. COVID-19 diagnostics include detection based on antibodies and nucleic acids, which are the most prevalent forms of COVID-19 testing. Nucleic acid-based tests involve sample collection from nasopharyngeal, oropharyngeal, or respiratory regions are collected and subjected to RT-PCR (Reverse Transcriptase PCR) [1]. In this process, SARS-CoV-2 RNA is converted to cDNA (complementary DNA), then primers specific to 3 loci of the viral cDNA are used for amplification [2]. The loci are ORF1ab which is the open reading frame of viral RDRP or RNA-Dependent RNA Polymerase, envelope protein or E gene, and nucleocapsid or N gene (Verma 2020). Either a singleplex or a multiplex format of RT-PCR is used.

A frequent problem reported in COVID-19 testing is falsenegatives, hence complementation of RT-PCR with CT scans is recommended for efficient diagnosis of SARS-CoV-2 infection [3,4]. An improved and efficient version of RT-PCR testing of COVID-19 is the targeting of RdRp/helicase (Hel), spike (S), and nucleocapsid (N) genes of SARS-CoV-2 [5].
In antibody-based testing of COVID-19, immunoglobulins IgG, IgM, and IgA are probed by ELISA (Enzyme-Linked ImmunoSorbent Assay). These immunoglobulins are produced by the body as a defense response to viral entry and detected after different number of days following COVID-19 infection [2]. In addition to diagnostics, several groups have generated panels of antibodies against spike or S protein of SARS-CoV-2 which binds to human receptor ACE2 [6]. The antibodies neutralize $S$ protein, hence preventing viral infection.

Another biotechnological advancement of COVID-19 testing is done by CRISPR-based methods where SARS-CoV-2 DNA Endonuclease-Targeted CRISPR Trans Reporter (DETECTR) uses CRISPR-Cas12 to detect COVID-19 [7]. Another COVID-19 detection process based on CRISPR uses recombinase-mediated polymerase pre-amplification of DNA or RNA which is a specific high-sensitivity enzymatic reporter unlocking (SHERLOCK) [8]. More methods based on CRISPR are developed to overcome the disadvantages of existing CRISPR-based tools for COVID-19 diagnosis.

In addition to the methods discussed above, there are other processes for COVID-19 diagnostics. Overall, a combination of biotechnological and bioengineering advances has led to the development of diagnostic and therapeutic interventions for COVID-19. Further research is needed to make them available to populations globally in a rapid, efficient, and inexpensive way to combat the pandemic.

\section{Acknowledgement}

None. 


\section{Conflicts of Interest}

No conflicts of interest.

\section{References}

1. Corman VM, Landt O, Kaiser M, Molenkamp R, Meijer A, et al. (2020) Detection of 2019 novel coronavirus (2019-nCoV) by real-time RT-PCR Euro Surveill 25(3).

2. Verma N, Patel D, Pandya A (2020) Emerging diagnostic tools for detection of COVID-19 and perspective. Biomed Microdevices 22(4): 83.

3. Ai T, Yang Z, Hou H, Zhan C, Chen C, et al. (2020) Correlation of Chest CT and RT-PCR Testing for Coronavirus Disease 2019 (COVID-19) in China: A Report of 1014 Cases. Radiology 296(2): E32-E40.

4. Fang Y, Zhang H, Xie J, Lin M, Ying L, et al. (2020) Sensitivity of Chest CT for COVID-19: Comparison to RT-PCR. Radiology 296(2): E115-E117.
5. Chan JF, Yip CC, To KK, Tang TH, Wong SC, et al. (2020) Improved Molecular Diagnosis of COVID-19 by the Novel, Highly Sensitive and Specific COVID-19-RdRp/Hel Real-Time Reverse Transcription-PCR Assay Validated In Vitro and with Clinical Specimens. J Clin Microbiol 58(5): e00310.

6. Wan J, Xing S, Ding L, Wang Y, Gu C, et al. (2020) Human-IgG-Neutralizing Monoclonal Antibodies Block the SARS-CoV-2 Infection. Cell Rep 32(3): 107918.

7. Broughton JP, Deng X, Yu G, Fasching CL, Servellita V, et al. (2020) CRISPR-Cas12-based detection of SARS-CoV-2. Nat Biotechnol 38(7): 870-874.

8. Kellner MJ, Koob JG, Gootenberg JS, Abudayyeh 00, Zhang F Sherlock (2019) Nucleic acid detection with CRISPR nucleases. Nat Protoc 14(10): 2986-3012. 